\title{
Identification of Ne VIII lines in H-deficient (pre-) white dwarfs: a new tool to constrain the temperature of the hottest stars ${ }^{\star \star \star}$
}

\author{
K. Werner ${ }^{1}$, T. Rauch ${ }^{1}$, and J. W. Kruk ${ }^{2}$ \\ 1 Institut für Astronomie und Astrophysik, Kepler Center for Astro and Particle Physics, Universität Tübingen, Sand 1, \\ 72076 Tübingen, Germany \\ e-mail: werner@astro.uni-tuebingen.de \\ 2 Department of Physics and Astronomy, Johns Hopkins University, Baltimore, MD 21218, USA
}

Received 25 June 2007 / Accepted 16 August 2007

\begin{abstract}
For the first time, we have identified Ne VIII absorption lines in far-UV spectra of the hottest known $\left(T_{\text {eff }} \gtrsim 150000 \mathrm{~K}\right)$ hydrogendeficient (pre-) white dwarfs of spectral type PG1159. They are of photospheric origin and can be matched by synthetic non-LTE line profiles. We also show that a number of UV and optical emission lines in these stars can be explained as being photospheric Ne VIII features and not, as hitherto suspected, as ultrahigh ionised O VIII lines created along shock-zones in the stellar wind. Consequently, we argue that the long-standing identification of the same emission lines in hot [WR]-type central stars as being due to ultrahigh-ionised species (O VII-VIII, C V-VI) must be revised. These lines can be entirely attributed to thermally excited species (Ne VII-VIII, N V, O VI). Photospheric Ne VIII lines are also identified in the hottest known He-rich white dwarf (KPD 0005+5106), some of which were also attributed to O VIII previously. This is a surprise because it must be concluded that KPD $0005+5106$ is much hotter $\left(T_{\text {eff }} \approx 200000 \mathrm{~K}\right)$ than hitherto assumed $\left(T_{\text {eff }} \approx 120000 \mathrm{~K}\right)$. This is confirmed by a re-assessment of the He II line spectrum. We speculate that the temperature is high enough to explain the mysterious, hard X-ray emission $(1 \mathrm{keV})$ as being of photospheric origin.
\end{abstract}

Key words. stars: abundances - stars: atmospheres - stars: evolution - stars: AGB and post-AGB - white dwarfs

\section{Introduction}

Observations of extremely hot post-AGB stars $\left(T_{\mathrm{eff}} \gtrsim 100000 \mathrm{~K}\right)$ with the Far Ultraviolet Spectroscopic Explorer (FUSE) have led to the discovery of many unidentified spectral lines. Their identification is difficult, mostly because of the lack of accurate atomic data for highly ionized elements. On the other hand, their identification is rewarding because they are often the only features by which particular species are accessible for abundance determinations. Such species can be used to test stellar evolutionary models. For example, several lines of fluorine (F V-VI), neon (Ne VII), silicon (Si V) and argon (Ar VII) were identified for the first time (Jahn et al. 2007; Werner et al. 2004a, 2005, 2007). In particular, the Ne VII $\lambda 973 \AA$ line occasionally displays a prominent $\mathrm{P}$ Cygni profile, which is an important tool for stellar wind analyses (Herald et al. 2005). In this paper, we report the identification of a number of Ne VIII lines in the FUSE spectra of the hottest known hydrogen-deficient (pre-) white dwarfs. We show that the mere occurrence of such highly ionized neon lines puts a strict lower limit on the effective temperature $(\approx 150000 \mathrm{~K})$.

\footnotetext{
* Based on observations made with the NASA-CNES-CSA Far Ultraviolet Spectroscopic Explorer. FUSE is operated for NASA by the Johns Hopkins University under NASA contract NAS5-32985.

$\star \star$ Some of the data presented in this paper were obtained from the Multimission Archive at the Space Telescope Science Institute (MAST). STScI is operated by the Association of Universities for Research in Astronomy, Inc., under NASA contract NAS5-26555. Support for MAST for non-HST data is provided by the NASA Office of Space Science via grant NAG5-7584 and by other grants and contracts.
}

Ne VIII is a one-valence electron, lithium-like ion with a relatively simple energy-term structure, however, a detailed compilation of accurate level energies and line wavelengths, which is a prerequisite of any quantitative work, became available only recently (Kramida \& Buchet-Poulizac 2006). Our model atmosphere and spectrum synthesis calculations predict the presence of Ne VIII lines in other wavelength regions, too. As a surprising result, we find that all previous identifications of ultra-high ionization (i.e., non-thermally excited) oxygen lines (O VIII) in UV and optical spectra of PG1159 stars, and the hottest known DO white dwarf, are wrong. We find that, instead, these lines are due to photospheric Ne VIII. The earlier identification of these lines as O VIII was motivated by the supposed occurrence of the same lines in early-type Wolf-Rayet central stars (i.e., spectral type [WCE]) and it was argued that they are formed in shocked wind regions. The results presented here provide evidence that also in [WCE] stars these, as well as other lines that were assigned to ultrahigh-ionized $\mathrm{C}$ and $\mathrm{O}$, do probably stem from thermally excited neon.

We present our observations and line identifications in Sect. 2 and describe our modeling in Sect. 3. The results from line profile fits to individual objects are presented in Sect. 4. Implications for [WCE] stars are discussed in Sect. 5 and we conclude with Sect. 6.

\section{Observations and Ne VIII line identifications}

FUSE observations and data reduction for most of our program stars were described in our previous work (Werner et al. 2004a,b, 2005, 2007). Table 1 lists our program stars with references to results from previous analyses. The FUSE instrument 
Table 1. Effective temperature and surface gravity of the program stars as taken from the literature. As described in the text, the discovery of Ne VIII lines suggests that $T_{\text {eff }}$ for KPD $0005+5106$ and Longmore 4 must be significantly higher.

\begin{tabular}{lcccc}
\hline \hline Object & $\begin{array}{c}\text { Spectral } \\
\text { type }\end{array}$ & $\begin{array}{c}T_{\text {eff }} \\
{[\mathrm{K}]}\end{array}$ & $\begin{array}{c}\log g \\
\text { (cgs) }\end{array}$ & Reference \\
\hline H1504+65 & PG1159 & 200000 & 8.0 & $\mathrm{~A}$ \\
RX J2117.1+3412 & PG1159 & 170000 & 6.0 & $\mathrm{~B}$ \\
NGC 246 & PG1159 & 150000 & 5.7 & $\mathrm{C}$ \\
K1-16 & PG1159 & 140000 & 6.4 & $\mathrm{D}$ \\
NGC 2371 & [WCE] & 135000 & 5.5 & $\mathrm{E}$ \\
Longmore 4 & PG1159 & 120000 & 5.5 & $\mathrm{~F}$ \\
KPD 0005+5106 & DO & 120000 & 7.0 & $\mathrm{G}$ \\
\hline
\end{tabular}

References: A: Werner etal. (2004b); B: Werner et al. (1996b); C: Rauch \& Werner (1997); D: Werner et al. (1992); E: Herald \& Bianchi (2004); F: Rauch \& Werner (1997); G: Werner et al. (1994).

consists of four coaligned telescopes, each with a prime-focus spectrograph. Descriptions of the FUSE instrument, and channel alignment and wavelength calibration issues, are given by Moos etal. (2000) and Sahnow etal. (2000). The FUSE spectra cover the wavelength range from the Lyman edge up to $1187 \AA$ with a spectral resolution of $0.05 \AA$. For spectra that are too faint to permit co-alignment of individual exposures, the spectral resolution may be degraded to $0.1 \AA$. The $\mathrm{S} / \mathrm{N}$ ratio in the LiF2a spectra in the vicinity of the Ne VIII lines being studied here ranges from a low of 17:1 per $0.05 \AA$ resolution element for Longmore 4 to $70: 1$ per resolution element for RX J2117.1+3412; it was greater than 45:1 for the other objects. The $\mathrm{S} / \mathrm{N}$ ratio in the LiF1b spectra at these wavelengths was typically $\sim 30 \%$ lower. UV spectra of KPD $0005+5106$ and RXJ2117.1+3412, taken with the FOS and GHRS spectrographs aboard the Hubble Space Telescope, were retrieved from the MAST archive. High-resolution optical spectra of K1-16, RX J2117.1+3412, KPD 0005+5106, and H1504+65 were obtained at the Keck observatory and the HIRES spectrograph. For details on data reduction see Zuckerman \& Reid (1998). Spectra of NGC 246 and Longmore 4 were obtained with the ESO Very Large Telescope and the UVES spectrograph, in the framework of the SPY project (Napiwotzki et al. 2003).

The starting point of our analysis was the identification of two strong and broad absorption features of Ne VIII at $\lambda=$ 1162-1165 A in the FUSE spectra of five PG1159 stars and the DO white dwarf KPD 0005+5106 (left panel of Fig. 1). They are blends of several $n=5 \rightarrow 6$ lines with high angular quantum number $l$. Ne VIII lines are not detected in the FUSE spectra of any other PG1159 star, indicating that the minimum $T_{\text {eff }}$ for exhibiting these lines is around $150000 \mathrm{~K}$ (this limit will be assessed more closely below). In addition to these lines, several weaker Ne VIII lines are detectable in the FUSE spectra of some objects, particularly in RX J2117.1+3412 (panels $a$ ) $-c$ ) in Fig. 2). These are low- $l n=5 \rightarrow 6$ lines and an isolated $n=6 \rightarrow 8$ line. The strongest, high- $l, n=6 \rightarrow 7$ transitions are located at $\lambda 1932 \AA$. We found only one archival spectrum covering this wavelength position, namely an HST/FOS observation of KPD $0005+5106$. An emission feature is barely detectable at this location (panel $d$ ) of Fig. 2), formerly attributed to O VIII $n=6 \rightarrow 7$ (Sion \& Downes 1992). We note that the line positions for O VIII and Ne VIII between levels with these high principal quantum numbers become indistinguishable. Both ions have the same core charge but their valence electrons are single $1 \mathrm{~s}$ and $2 \mathrm{~s}$ electrons, respectively. Based on our model computations described below, we attribute the previously discovered emission features at $\lambda \lambda 2977,4340$, and $6068 \AA$ (Figs. 2 and 1) to the $n=7 \rightarrow 8, n=8 \rightarrow 9$, and $n=9 \rightarrow 10$ transitions of Ne VIII, respectively. They, too, were previously thought to stem from O VIII (Werner \& Heber 1992; Werner et al. 1996). The $\lambda 6068 \AA$ emission line was also detected in the very hot PG1159 stars Longmore 3 and HE 1429-1209 (Werner et al. 1994, 2004a).

Table 2 summarizes all Ne VIII lines that were detected in any of the examined objects. The NIST ${ }^{1}$ wavelengths coincide with the photospheric rest wavelengths, with the exception of the $5 \mathrm{p} \rightarrow 6 \mathrm{~d}, 5 \mathrm{f} \rightarrow 6 \mathrm{~d}$, and $6 \mathrm{p} \rightarrow 8 \mathrm{~d}$ transitions. Their observed wavelengths are smaller by $0.16,0.09$, and $0.07 \AA$, respectively. This is corrected for in panels $b$ ) and $c$ ) of Fig. 2 and in Table 2.

We note that we have not detected any Ne VIII lines in the hottest $\left(T_{\text {eff }} \approx 100000 \mathrm{~K}\right)$ hydrogen-rich central stars or DA white dwarfs from which FUSE spectra are available. The reason is that their temperature is not sufficiently high (all have $T_{\text {eff }}<$ $150000 \mathrm{~K}$ ). The same holds for all hot DO white dwarfs besides KPD 0005+5106. The possible presence of Ne VIII lines in the FUSE spectra of [WCE] stars is discussed below (Sect. 5).

\section{Model atmospheres and neon line formation calculations}

We use a grid of line-blanketed non-LTE model atmospheres, which is described in detail in Werner et al. (2004a). In essence, the models include the main photospheric constituents, namely, $\mathrm{He}, \mathrm{C}, \mathrm{O}$, and $\mathrm{Ne}$. The neon model atom consists of the ionization stages Ne IV-IX. Of particular importance for the investigations presented in this paper is the Ne VIII ion. It consists of 5 NLTE levels and 6 lines, which is sufficient for the atmospheric model structure computation, but considerable extensions were required in order to be able to compute profiles of the identified highly excited lines. Since we aimed at the computation of lines involving the $n=10$ levels (the $n=9 \rightarrow 10$ emission at $6068 \AA$ ), we included all 77 levels up to $n=12$ with all 511 line transitions between them. In addition, levels up to $n=14$ are included as LTE levels. With this extended Ne VIII model ion, we performed line-formation iterations for neon, i.e., keeping the atmospheric structure fixed. For the final spectrum synthesis, fine-structure splitting of levels and lines was considered, if possible. Energies for fine-structure splitting are not available for all levels. In particular, they are only partly available for levels involved in the $n=5 \rightarrow 6$ absorption lines at $\lambda 1162-1165 \AA$. As a consequence, the detailed structure of the computed line profile cores within these absorption troughs cannot exactly match the observations and, indeed, a close inspection of Fig. 1 reveals this problem. Level energies were taken from NIST (Kramida \& Buchet-Poulizac 2006). Oscillator strengths were taken from the Opacity (Seaton et al. 1994) and IRON (Hummer et al. 1993) Projects databases (TIPTOPbase ${ }^{2}$ ), which are, however, not complete for all transitions of our model atom. For lines involving levels with $n=11$ and 12, we extrapolated oscillator strengths from transitions to lower quantum numbers. For a lithium-like ion this appears reasonably accurate; the estimates are probably within a $10 \%$ error. Photoionization cross-sections are taken from the Opacity Project database when available or, otherwise, computed in hydrogen-like approximation. Electron collisional rates were calculated with usual approximation formulae. The Ne model atoms that were used for this analysis have

\footnotetext{
1 http://physics.nist.gov/PhysRefData/ASD/index.html

2 http://vizier.u-strasbg.fr/topbase/
} 

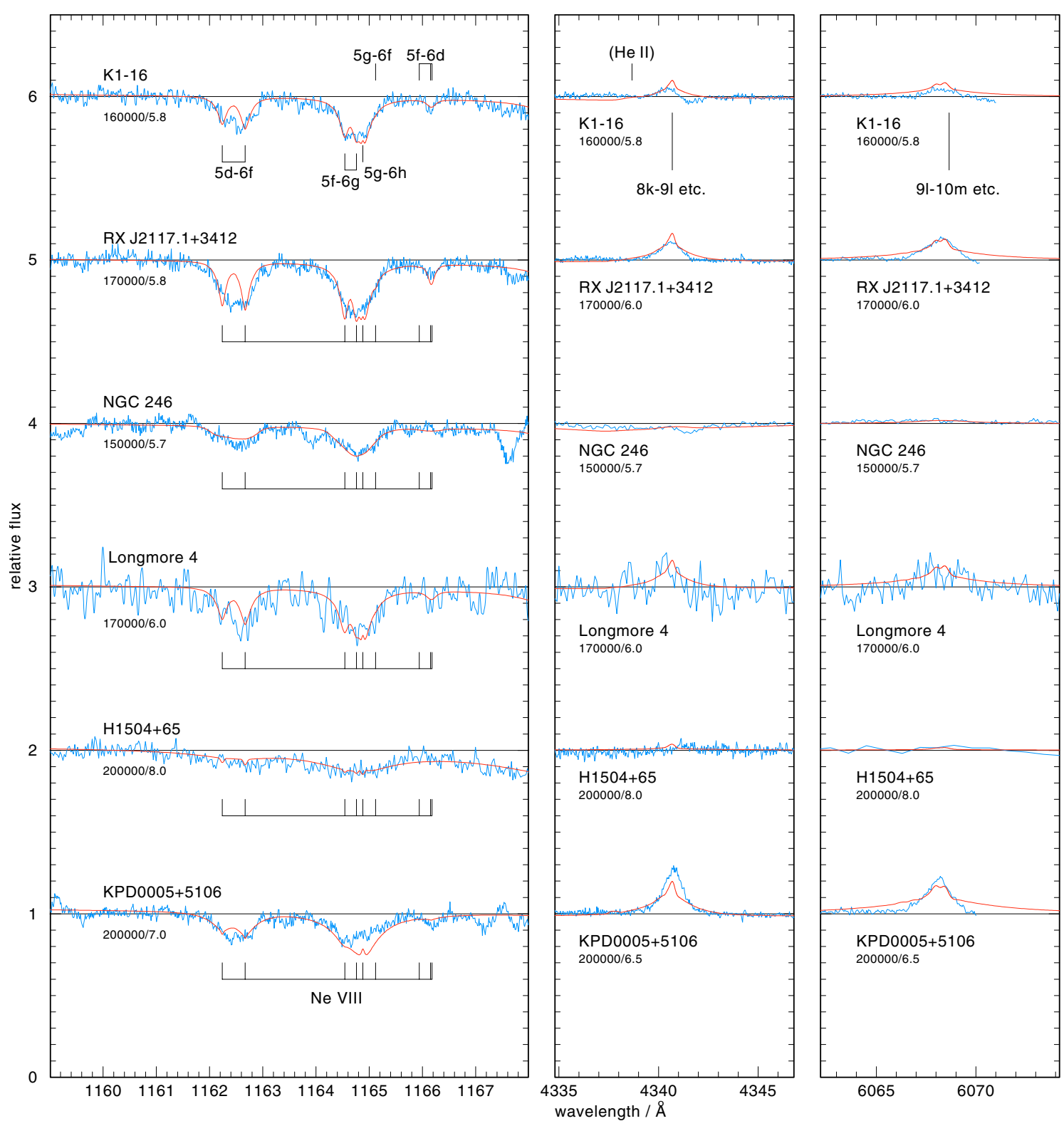

Fig. 1. Left panel: identification of Ne VIII lines in the FUSE spectra of PG1159 stars and the DO white dwarf KPD 0005+5106. Several lines of the $n=5 \rightarrow 6$ transition are detected, as labeled in detail at the uppermost spectrum. Overplotted are computed profiles with $T_{\text {eff }}$ and log $g$, as indicated. Middle and right panels: optical spectral regions where we identified the Ne VIII $n=8 \rightarrow 9$ and $n=9 \rightarrow 10$ transitions. All observed spectra are shifted such that the photospheric lines appear at their rest wavelengths.

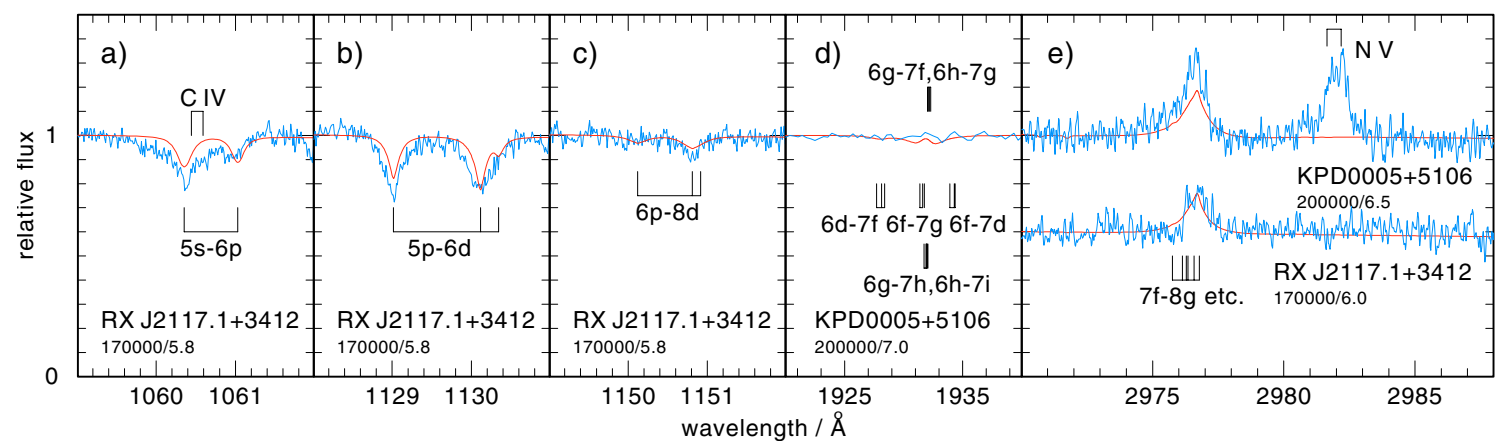

Fig. 2. Details of other spectral regions of the PG1159-type central star RX J2117.1+3412 and the DO white dwarf KPD $0005+5106$ displaying Ne VIII lines. Panels a)-c) show $n=5 \rightarrow 6$ and $n=6 \rightarrow 8$ transitions (the blending C IV line in panel a) is not included in the model). Panel d) shows a barely detectable $n=6 \rightarrow 7$ emission feature in the HST/FOS spectrum of KPD 0005+5106, which was previously assigned to O vIII. Panel e) displays HST/GHRS spectra with a Ne VIII $n=7 \rightarrow 8$ emission line that was also thought to stem from O VIII. The adjacent emission feature in KPD 0005+5106 is from nitrogen; it is not included in the model. 
Table 2. Ne VIII lines identified in our program stars. Wavelengths are computed from NIST level energies (except for the $5 \mathrm{p} \rightarrow 6 \mathrm{~d}, 5 \mathrm{f} \rightarrow 6 \mathrm{~d}$, and $6 \mathrm{p} \rightarrow 8 \mathrm{~d}$ transitions, see text) and given in vacuum or air for $\lambda$ smaller or larger than $3000 \AA$, respectively. The presence of the feature at $\lambda 1932 \AA$ is uncertain.

\begin{tabular}{ll}
\hline \hline Wavelength $/ \AA$ & Transition \\
\hline $1060.36,1061.03$ & $5 \mathrm{~s} \rightarrow 6 \mathrm{p}$ \\
$1129.02,1130.12,1130.35$ & $5 \mathrm{p} \rightarrow 6 \mathrm{~d}$ \\
$1150.12,1150.81,1150.92$ & $6 \mathrm{p} \rightarrow 8 \mathrm{~d}$ \\
$1162.24,1162.67$ & $5 \mathrm{~d} \rightarrow 6 \mathrm{f}$ \\
$1164.54,1164.75$ & $5 \mathrm{f} \rightarrow 6 \mathrm{~g}$ \\
1164.88 & $5 \mathrm{~g} \rightarrow 6 \mathrm{~h}$ \\
$1165.94,1166.15,1166.18$ & $5 \mathrm{f} \rightarrow 6 \mathrm{~d}$ \\
$1170.04,1170.29$ & $6 \mathrm{~d} \rightarrow 8 \mathrm{f}$ \\
$(1932.04)$ & $6 \mathrm{~h} \rightarrow 7 \mathrm{i}$ etc. \\
2976.75 & $7 \mathrm{i} \rightarrow 8 \mathrm{k}$ etc. \\
4340.77 & $8 \mathrm{k} \rightarrow 91$ etc. \\
6068.63 & $9 \mathrm{l} \rightarrow 10 \mathrm{~m}$ etc. \\
\hline
\end{tabular}

been developed in the framework of the German Astrophysical Virtual Observatory $\left(\mathrm{GAVO}^{3}\right)$ project and are provided within the Tübingen Model-Atom Database TMAD ${ }^{4}$.

The treatment of Stark broadening of Ne VIII lines poses a severe problem. We employ an approximate formula to compute Stark widths, which we use routinely to compute profiles of lines between highly excited levels of lithium-like ions (C IV, O VI; Werner et al. 1991), and which accounts for linear Stark broadening. While this formula gave reasonable results for the other Li-isoelectronic ions in our past work, we found that the $n=5 \rightarrow 6$ absorption lines in the FUSE spectral range came out much too narrow (by about a factor of three) compared to all of the observations. Interestingly, a similar notorious problem is encountered in the interpretation of Ne VIII line widths of laboratory spectra. Measurements of the $3 \mathrm{~s} \rightarrow 3 \mathrm{p}$ transition at $2821 \AA$ (Glenzer et al. 1992) display line profiles that are roughly a factor of two wider than predicted by theory, i.e., the $Z^{-2}$ scaling of the line width, as predicted by the impact theory, significantly deviates from observations. Even today, this discrepancy is not resolved, despite of intense efforts with both improved experiments and quantum-mechanical calculations (Hegazy et al. 2003; Griem \& Ralchenko 2006). Our finding from stellar spectra indirectly suggests that the line widths measured in laboratory are in fact correct, and that instead, the line-broadening theory is still inaccurate. It is necessary to note, however, that we observe different lines of Ne VIII.

To cope with this problem we formally reduced the ionic core charge of Ne VIII in our line broadening formula from $Z=8$ to $Z=2$. This yields widths of the $n=5 \rightarrow 6$ lines that coincide with the observations. Of course, this procedure is rather unsatisfactory and would prohibit an accurate abundance determination from these lines. In addition, a close inspection of the computed profiles for the optical lines (i.e., the higher- $n$ transitions, Fig. 1) reveals that they become too broad with our crude $Z$-reduction. Fortunately, the neon abundance of all PG1159 stars discussed here is known to be of the order $2 \%$ (Werner et al. 2004a). The focus of our paper is on the extreme temperature sensitivity of the Ne VIII lines and line broadening is a subordinate effect only.

\footnotetext{
3 http://www.g-vo.org

${ }^{4}$ http://astro.uni-tuebingen.de/ rauch/TMAD/TMAD .html
}

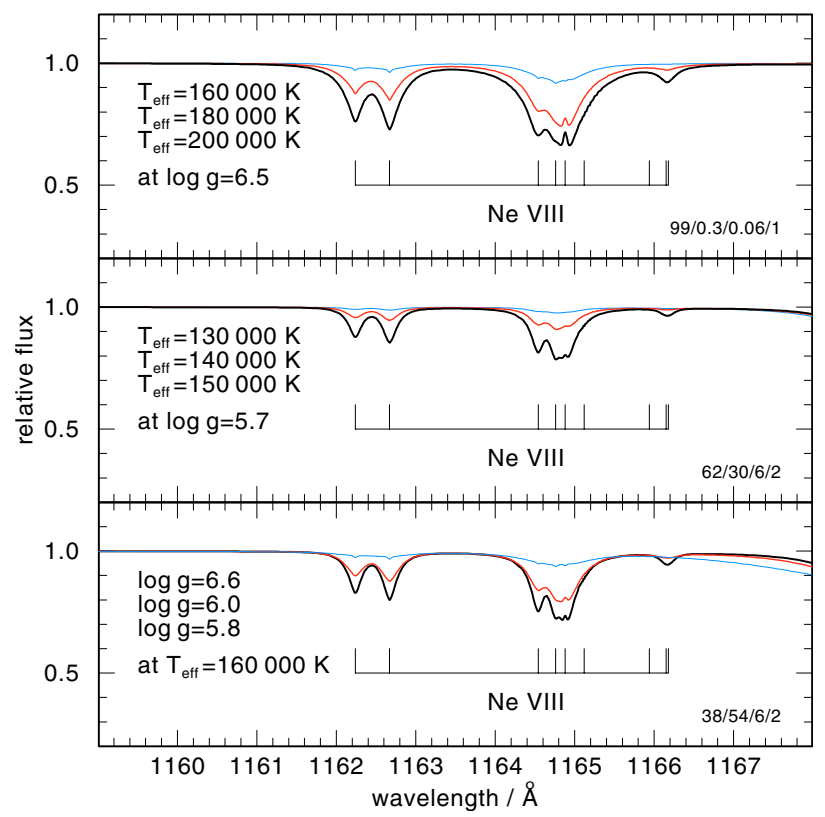

Fig. 3. The Ne VIII lines are strongly sensitive to $T_{\text {eff }}$ and $\log g$. Generally, they become stronger with increasing $T_{\text {eff }}$ and decreasing $\log g$. Top panel: $T_{\text {eff-sensitivity in DO models with } \log g=6.5 \text {. The }}$ lines disappear if $T_{\text {eff }}$ is below $160000 \mathrm{~K}$. Middle panel: $T_{\text {eff }}$-sensitivity in PG1159 models with lower gravity $(\log g=5.7)$. In this case, the lines are detectable down to $T_{\text {eff }}=140000 \mathrm{~K}$. Bottom panel: $\log g$ sensitivity in PG1159 models at $T_{\text {eff }}=160000 \mathrm{~K}$. The gravity must be sufficiently low, otherwise the Ne VIII lines are not detectable. The numbers in the lower right corner of the panels give the model abundances of $\mathrm{He} / \mathrm{C} / \mathrm{O} / \mathrm{Ne}$ in $\%$ mass fraction.

\section{Results}

We now compare our computed Ne VIII line profiles to the observations (Fig. 1). It is instructive to look at Fig. 3, in which we demonstrate that the strengths of the $\lambda \lambda 1162-1165 \AA$ absorption lines strongly depend on $T_{\text {eff }}$ and $\log g$. The minimum $T_{\text {eff }}$ for a line detection is a strong function of gravity, ranging from about $160000 \mathrm{~K}$ at $\log g=6.5$ to $140000 \mathrm{~K}$ at $\log g=5.7$. The mere detection of these Ne VIII lines thus provides a strict lower limit to $T_{\text {eff }}$. Generally, the overall characteristics of the Ne VIII lines are reproduced by the models. We find that the UV lines are in absorption, while the optical lines are in emission.

Let us discuss in some detail each of the six objects in which we detected the UV Ne VIII lines, particularly those cases in which contradictions to $T_{\text {eff }}$ determinations from previous work appear. This mainly affects the DO white dwarf KPD 0005+5106, whereas for the PG1159 stars the Ne VIII line features can be matched with model parameters that are in good or acceptable agreement with previous results.

\subsection{PG1159 stars}

Models for individual objects were computed with element abundances as given in Werner et al. (2005). As already mentioned, the neon abundance is kept fixed at $2 \%$. We started with values for $T_{\text {eff }}$ and $\log g$ also taken from this reference; typical uncertainties are $10 \%$ and 0.5 dex, respectively.

K1-16 The previously determined parameters are $T_{\text {eff }}=$ $140000 \mathrm{~K}$ and $\log g=6.4$. Clearly, a model with these values 

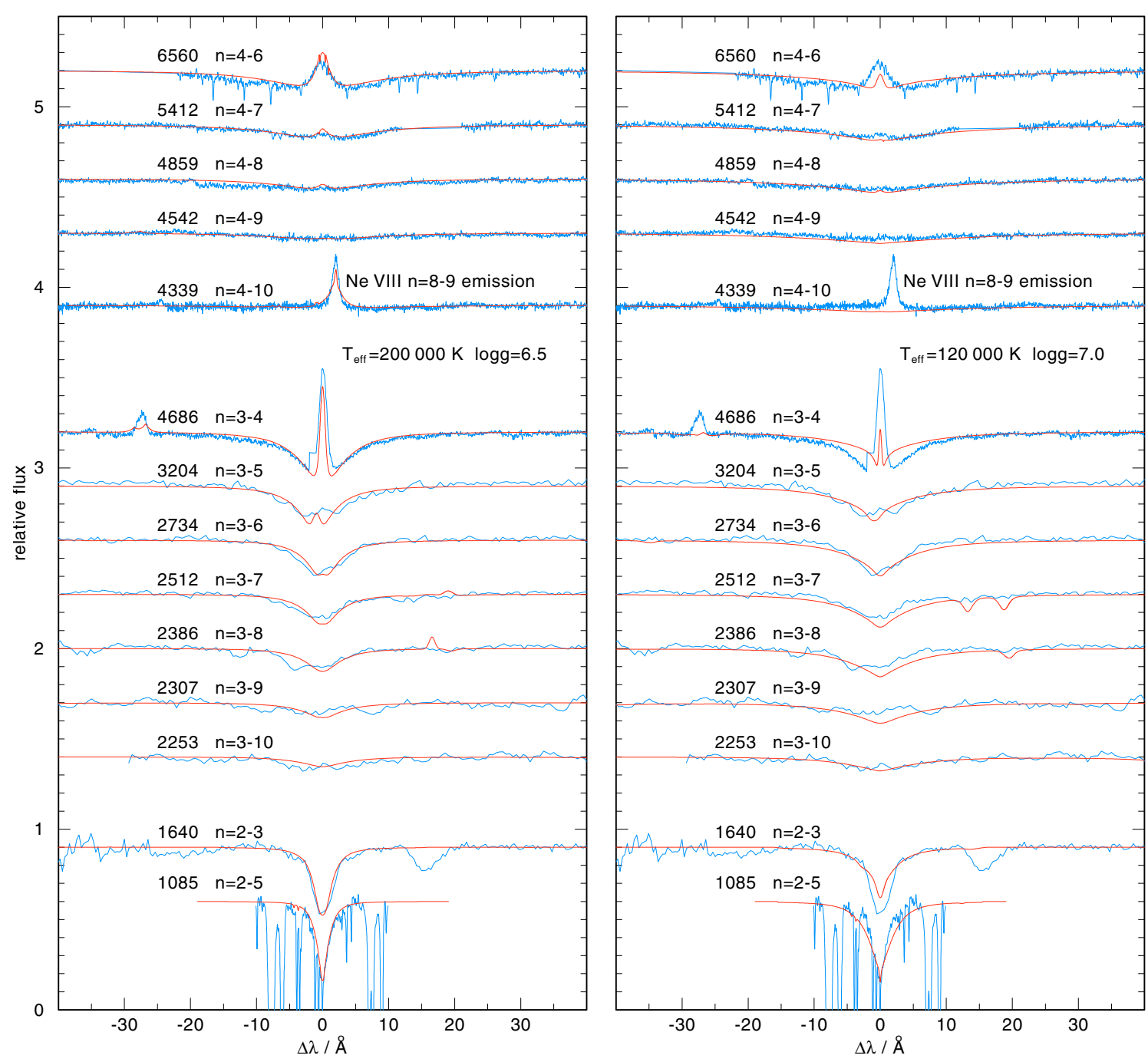

Fig. 4. The He II lines in KPD $0005+5106$ are compared with two different models. Left panel: $T_{\text {eff }}=200000 \mathrm{~K}, \log g=6.5$. Right panel: $T_{\text {eff }}=$ $120000 \mathrm{~K}, \log g=7.0$. The hotter model yields a much better fit. In particular, only this model matches the emission cores in the $\lambda \lambda 6560$ and $4686 \AA$ lines. The emission line at $\lambda 4339 \AA$ is due to Ne VIII and is only exhibited in the hotter model.

shows no Ne VIII lines at all. We find an acceptable fit only at $T_{\text {eff }}$ and $\log g$ values that are slightly beyond the error bars, namely $T_{\text {eff }}=160000 \mathrm{~K}$ and $\log g=5.8$.

RX J2117.1+3412 We find a good fit to the Ne VIII lines with models close to the literature values $\left(T_{\text {eff }}=170000 \mathrm{~K}, \log g=\right.$ 6.0). At these parameter values, the Ne VIII absorption lines are strongest. This explains why we can identify a number of additional weak Ne VIII lines in the FUSE spectrum (Fig. 2).

NGC 246 A good fit to the Ne VIII lines is obtained at the literature values $\left(T_{\text {eff }}=150000 \mathrm{~K}, \log g=5.7\right)$. Note that NGC 246 is a fast rotator and the computed profiles were broadened with $70 \mathrm{~km} \mathrm{~s}^{-1}$ (Rauch \& Werner 1997).

Longmore 4 The strong Ne VIII lines in the FUSE spectrum suggest that this star is as hot as RX $2117.1+3412$. We find a good fit with a $T_{\text {eff }}=170000 \mathrm{~K}, \log g=6.0$ model. Therefore, Longmore 4 is significantly hotter than previously thought $\left(T_{\text {eff }}=120000 \mathrm{~K}, \log g=5.5\right)$. This result is, however, not surprising because we have already found independent hints that the temperature is at least about $150000 \mathrm{~K}$ (Werner et al. 2004a). Note that Longmore 4 exhibited much more prominent optical Ne VIII lines during its observed "outburst" and change of spectral type from PG1159 to [WCE] (Werner et al. 1992).

H1504+65 The Ne VIII lines in the FUSE spectrum are shallow and broad. This is a consequence of the high gravity (8.0). The profiles confirm the extremely high $T_{\text {eff }}(200000 \mathrm{~K})$.

\subsection{The DO white dwarf KPD $0005+5106$}

An analysis of optical and HST UV spectra of KPD $0005+5106$ gave the result $T_{\text {eff }}=120000 \mathrm{~K}$ and $\log g=7$ (Werner et al. 1994). Although being the hottest known DO white dwarf, the identification of Ne VIII lines is a big surprise. Figure 3 shows that at this relatively high gravity the temperature must be around $180000 \mathrm{~K}$. We find that models with $T_{\text {eff }}=180000-200000 \mathrm{~K}$ and $\log g=6.5-7.0$ give the best fit to the Ne VIII lines. Interestingly, this is not in contradiction with the He II lines. We even find that a model with $T_{\text {eff }}=200000 \mathrm{~K}$ and $\log g=6.5$ gives better He II line fits than the cooler $120000 \mathrm{~K}$ model. This is shown in Fig. 4. To be more specific, the hot model is able to 
match the height of the central emissions reversals in the $\lambda \lambda 4686$ and $6560 \AA$ line cores. At the same time, the $\lambda 1640 \AA$ line is matched very well. We conclude that KPD $0005+5106$ is significantly hotter than previously thought.

Unlike for the PG1159 stars, the neon abundance in KPD $0005+5106$ is not known from previous analyses. The usual diagnostic lines are Ne VII $\lambda \lambda 973$ and $3644 \AA$ A. The FUSE spectrum of KPD $0005+5106$ is contaminated by interstellar $\mathrm{H}_{2}$ absorption, making the detection of Ne VII $\lambda 973 \AA$ impossible. In a medium-resolution optical spectrum published by Werner et al. (1994), no line feature is detected at $\lambda 3644 \AA$. We have verified that this is compatible with all our models presented here in the $T_{\text {eff }}=120000-200000 \mathrm{~K}$ range.

We assumed that the $\mathrm{Ne}$ abundance is determined by radiative levitation. The hottest model presented by Chayer et al. (1995) has $T_{\text {eff }}=100000 \mathrm{~K}$ with $\log g=7.5$ and predicts $\log (\mathrm{Ne} / \mathrm{He})=-4$ by number. It is difficult to extrapolate this to a significantly higher $T_{\text {eff }}$ but the tendency is that the Ne abundance increases with $T_{\text {eff }}$. A further increase can be expected because of the smaller gravity. Although uncertain, it is not unreasonable that we set $\mathrm{Ne}=1 \%$ (by mass), that is, $\log (\mathrm{Ne} / \mathrm{He})=$ -2.7 by number. We stress that the high $T_{\text {eff }}$ derived from the $\mathrm{Ne}$ VIII lines is hardly affected by this assumption. In addition, the He II line profiles are essentially independent of the neon abundance.

\section{Implications for ultrahigh-ionisation emission line identifications in [WCE] stars}

Since the supposed identification of several optical emission lines in the hot [WC] star Sand 3 as being due to ultrahigh ionised (i.e. non-thermally excited) $\mathrm{C}$ and $\mathrm{O}$ (C V and O VII-VIII) (Barlow et al. 1980), a number of authors claimed the identification of these features in other early-type [WC] stars. In the light of our results, we propose that most, and probably all, of these features stem from thermally excited ionisation stages of neon (Ne VII-VIII), carbon and oxygen. Table 3 summarizes our proposed identifications. We give qualitative arguments for this, but detailed NLTE modeling with expanding model atmospheres will be necessary for a quantitative confirmation.

A broad emission feature in Sand 3 and other [WCE]s at $\lambda 3893 \AA$ was attributed to the $n=7 \rightarrow 8$ transition of O VII. We propose that it originates from a prominent Ne VII multiplet recently identified in the hottest PG1159 central stars (Werner et al. 2004a), which has its strongest component located at $\lambda 3892 \AA$. In addition, the Ne VII $n=7 \rightarrow 8$ transition contributes. The emission at $\lambda 4340 \AA$ is probably from Ne VIII, not O VIII. Emission lines at $\lambda \lambda 4555 \AA$ and $5665 \AA$ were assigned to the O VII transitions $n=9 \rightarrow 11$ and $n=8 \rightarrow 9$, respectively. We suggest that they are lines between high Rydberg states of $\mathrm{Ne}$ VII, with the same principal quantum numbers (level energies are given in NIST and Lapierre \& Knystautas 1999). This is supported by our discovery of two high- $l n=8 \rightarrow 9$ emission lines of Ne VII at $\lambda 5665 \AA$ in some of our program stars (Fig. 5). Finally, the strong emission feature at $\lambda 4945 \AA$ was assigned to $\mathrm{CV} n=7 \rightarrow 6$. We think that it is the respective $\mathrm{N} V$ transition, as in the case of KPD $0005+5106$. Nitrogen is definitely present in Sand 3 , because the $3 \mathrm{~s} \rightarrow 3 \mathrm{p}$ emission doublet at $\lambda \lambda 4602,4620 \AA$ is present, as well as a prominent P Cygni profile of the $\mathrm{N} v$ resonance line. Thus, the entire optical emission line spectrum of Sand 3 and other [WCE]s can be explained without invoking ultrahigh-ionisation features.
Table 3. List of lines for which we propose photospheric identifications as opposed to previously thought identifications as non-photospheric ultrahigh-ionisation features in PG1159 stars, [WCE] stars, and in the DO KPD 0005+5106. The O VI and N v emission lines were identified before but thought to be blended by the ultrahigh ionisation features.

\begin{tabular}{|c|c|c|}
\hline Wavelength / $\AA$ & $\begin{array}{l}\text { Old ultrahigh- } \\
\text { ionisation } \\
\text { identification }\end{array}$ & $\begin{array}{l}\text { New photospheric } \\
\text { identification }\end{array}$ \\
\hline 1932 & O VIII $\quad n=7 \rightarrow 8$ & $n=7 \rightarrow 8$ \\
\hline 2977 & $n=6 \rightarrow 7$ & Ne VIII $n=6 \rightarrow 7$ \\
\hline 3893 & $n=7 \rightarrow 8$ & $\begin{array}{l}\text { Ne VII } 3 \mathrm{p}^{3} \mathrm{P}^{\mathrm{o}} \rightarrow 3 \mathrm{~d}^{3} \mathrm{D} \\
\text { plus Ne VII } n=7 \rightarrow 8\end{array}$ \\
\hline 4340 & $n=8 \rightarrow 9$ & Ne VIII $n=8 \rightarrow 9$ \\
\hline 4500 & $\mathrm{C} \mathrm{VI} \quad n=8 \rightarrow 10$ & O VI $\quad n=8 \rightarrow 10$ \\
\hline 4555 & $n=9 \rightarrow 11$ & Ne VII $n=9 \rightarrow 11$ \\
\hline 4945 & $\mathrm{CV} \quad n=6 \rightarrow 7$ & $\mathrm{Nv} \quad n=6 \rightarrow 7$ \\
\hline 5290 & C VI $n=7 \rightarrow 8$ & O VI $n=7 \rightarrow 8$ \\
\hline 5665 & O VII $\quad n=8 \rightarrow 9$ & Ne VII $n=8 \rightarrow 9$ \\
\hline 6068 & O VIII $\quad n=9 \rightarrow 10$ & Ne VIII $n=9 \rightarrow 10$ \\
\hline
\end{tabular}

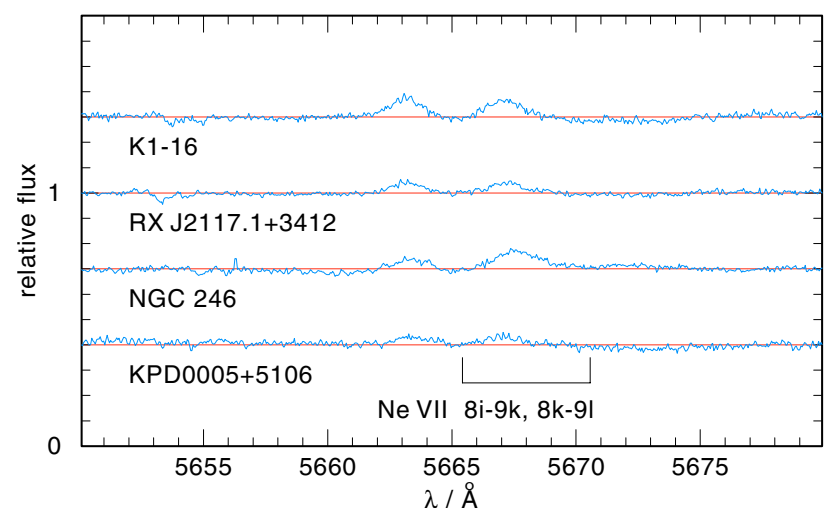

Fig. 5. First identification of two high- $l n=8 \rightarrow 9$ emission lines of Ne VII (!). A strong and broad emission feature is seen in [WCE] stars at this location. We propose that it is due to these Ne VII transitions and not due to ultrahigh ionized O VII. The labels denote the theoretical line positions using energy levels that are not precisely known.

Based on observations with the International Ultraviolet Explorer (IUE), several papers appeared in the literature claiming the presence of ultrahigh ionisation lines in the UV spectra of [WCE] stars. In Sand 3, as well as in NGC 5315 and NGC 6905, Feibelman (1996a,b) discovered the $\lambda \lambda 1932 \AA$ and $2977 \AA$ emission features that we have discussed above, and he also assigned them to O VIII. As in KPD 0005+5106 and RX J2117.1+3412 (Fig. 2), they probably are from Ne VIII.

Feibelman (1996b) even claimed the existence of an "O VIII sequence" of planetary-nebulae nuclei, mainly based on the supposed O VII-VIII identifications in PG1159 and [WCE] stars. Obviously, that idea must now be discarded. This is further corroborated by the fact that we detected the Ne VIII lines at $\lambda 1162-1165 \AA$ in a [WCE] star too, namely in NGC 2371 (Fig. 6). The line strengths suggest $T_{\text {eff }} \approx 150000 \mathrm{~K}$, which is, considering error limits, in good agreement with the result of a detailed analysis of FUSE and IUE spectra $(135000 \mathrm{~K}$; Herald \& Bianchi 2004). NGC 2371 was classified as [WC4] by Acker \& Neiner (2003). These authors have based a new spectral classification system for the hottest [WC]s on the occurrence of O VII-VIII lines in the optical spectrum. Our identification as Ne VII-VIII lines means that the empirical classification criteria remain essentially correct because only the hottest [WC]s are able to show these lines. 


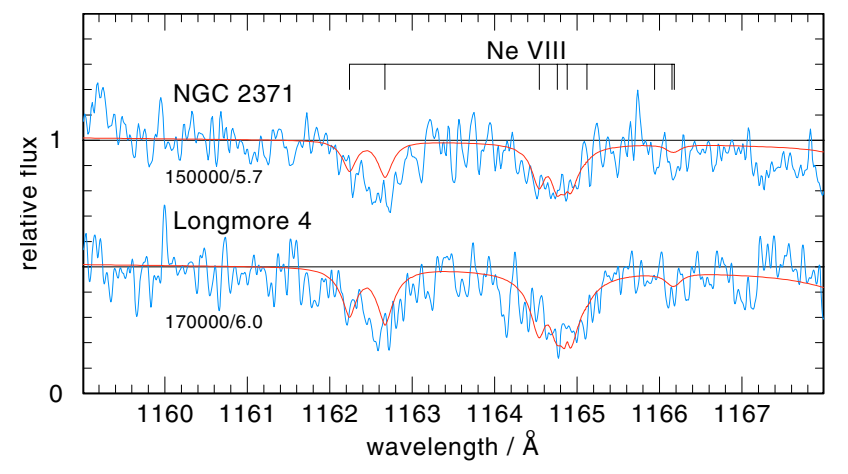

Fig. 6. Identification of Ne VIII lines in the [WCE] central star NGC 2371 (top) and comparison to the PG1159-type central star Longmore 4 (bottom).

\section{Summary and conclusions}

We have identified Ne VIII absorption lines in FUSE spectra of PG1159 stars and a DO white dwarf. Line profile fits confirm that the PG1159 stars are the very hottest members of their spectral class $\left(T_{\text {eff }} \geq 150000 \mathrm{~K}\right)$. We have shown that two wellknown emission lines in the optical spectra of these stars are also from Ne VIII and not, as previously thought, from O VIII. While Ne VIII is thermally excited in the hot photospheres, the existence of $\mathrm{O}$ VIII would require temperatures of the order of one million $\mathrm{K}$, thus, an unknown process (e.g., shock zones in the wind) was invoked to explain these emission lines. This is no longer necessary.

We argue that these, and probably all, emission lines in earlytype [WC] central stars that are usually assigned to ultrahighionisation stages (O VII-VIII, C V) originate from Ne VII-VIII and other thermally excited species.

The discovery of Ne VIII lines in the central star Longmore 4 suggests that its $T_{\text {eff }}$ is distinctively higher than previously thought $(170000 \mathrm{~K}$ instead of $120000 \mathrm{~K})$. To a smaller extent, the same tendency is seen for the PG1159 star K1-16 and the [WCE] star NGC 2371. This calls for a re-analysis of the complete spectra of these stars to see how the fit to other line features can be reconciled. The computation of model grids with varying parameters $T_{\text {eff }}, \log g$, and abundances of the most important elements $(\mathrm{He}, \mathrm{C}, \mathrm{O}, \mathrm{Ne})$ will be necessary. Since the stars display prominent $\mathrm{P}$ Cygni line profiles, it could be advantageous to use expanding atmosphere models with the available spectral data. As already mentioned, the neon abundance cannot be determined from the newly discovered Ne VIII lines because of the uncertainties with the line broadening theory. Instead, the strong P Cygni profile of the Ne VII $\lambda 973 \AA$ line exhibited by these stars can be used as a sensitive tool (Bianchi \& Herald 2007). Again, detailed parameter studies must be performed to find which neon abundance and which mass-loss rate yield good fits to this Ne VII line profile, while at the same time determining the $T_{\text {eff }}$ of the model that is high enough to produce detectable Ne VIII lines.

A surprising result of our investigation is the identification of Ne VIII lines in the hottest known DO white dwarf, KPD $0005+5106$. We conclude that its temperature is close to $T_{\text {eff }}=200000 \mathrm{~K}$ and, hence, that it is significantly hotter than hitherto thought $(120000 \mathrm{~K})$. As in the case of the PG1159 stars, the optical Ne VIII emission lines were previously assigned to ultrahigh-ionized O VIII. The announcement of the discovery of a relatively soft X-ray emitting corona about
KPD 0005+5106 seemed to support this assignment (Fleming et al. 1993). However, the analysis of a Chandra spectrum exhibiting flux in the range $20-80 \AA$ proved that the soft X-rays stem from the photosphere of KPD 0005+5106 (Drake \& Werner 2005). The deposition of the corona is now in accordance with the deposition of the ultrahigh-ionisation lines. A comprehensive re-analysis of all available data (Chandra, FUSE, HST, Keck) is required to tightly constrain the atmospheric parameters and metal abundances.

It remains to be seen how the extremely high effective temperature derived in our work relates to the observed hard X-ray emission at $1 \mathrm{keV}(12 \AA$ ) from KPD 0005+5106 (O'Dwyer et al. 2003). It might be that this emission is also of photospheric origin. After all, the entire spectral properties of KPD 0005+5106 could be deciphered as thermal photospheric radiation. In any case, the star has turned out to be by far the hottest known DO white dwarf.

Acknowledgements. We are grateful to the referee, Luciana Bianchi, for a careful reading of the manuscript and for her constructive comments. We thank Uli Heber and Ralf Napiwotzki for putting their Keck and VLT spectra at our disposal. We thank A.E. Kramida, H.R. Griem, H.-J. Kunze, M.S. Dimitrijević, and É. Knystautas for discussions on atomic data and sending us reprints. T.R. is supported by the German Astrophysical Virtual Observatory project of the German Federal Ministry of Education and Research under grant 05 AC6VTB. J.W.K. is supported by the FUSE project, funded by NASA contract NAS5-32985.

\section{References}

Acker, A., \& Neiner, C. 2003, A\&A, 403, 659

Barlow, M. J., Blades, J. C., \& Hummer, D. G. 1980, ApJ, 241, L27

Bianchi, L., \& Herald, J. E. 2007, Proceedings, IAU General Assembly 2006, Joint Discussion 04, in press

Chayer, P., Fontaine, G., \& Wesemael, F. 1995, ApJS, 99, 189

Drake, J. J., \& Werner, K. 2005, ApJ, 625, 973

Feibelman, W. A. 1996a, ApJ, 464, 910

Feibelman, W. A. 1996b, PASP, 108, 664

Fleming, T. A., Werner, K., \& Barstow, M. A. 1993, ApJ, 416, L79

Glenzer, S., Uzelac, N. I., \& Kunze, H.-J. 1992, Phys. Rev. A, 45, 8795

Griem, H. R., \& Ralchenko, Y. 2006, in Spectral Line Shapes: 18th International Conference, ed. E. Oks, \& M. Pindzola, American Institute of Physics, CP874, 14

Hegazy, H., Seidel, S., Wrubel, T., \& Kunze, H.-J. 2003, JQSRT, 81, 221

Herald, J. E., \& Bianchi, L. 2004, ApJ, 609, 378

Herald, J. E., Bianchi, L., \& Hillier, D. J. 2005, ApJ, 627, 424

Hummer, D. G., Berrington, K. A., Eissner, W., et al. 1993, A\&A, 279, 298

Jahn, D., Rauch, T., Reiff, E., et al. 2007, A\&A, 462, 281

Kramida, A. E., \& Buchet-Poulizac, M.-C. 2006, Eur. Phys. J. D, 39, 173

Lapierre, A., \& Knystautas, É. J. 1999, Phys. Scr., 59, 426

Moos, H. W., Cash, W. C., Cowie, L. L., et al. 2000, ApJ, 538, L1

Napiwotzki, R., Christlieb, N., Drechsel, H., et al. 2003, The Messenger, 112, 25 O’Dwyer, I. J., Chu, Y.-H., Gruendl, R. A., Guerrero, M. A., \& Webbink, R. F. 2003, AJ, 125, 2239

Rauch, T., \& Werner, K. 1997, in The Third Conference on Faint Blue Stars, ed. A. G. D. Philip, J. Liebert, \& R. A. Saffer (Schenectady, NY: L. Davis Press), 217

Sahnow, D. J., Moos, H. W., \& Ake, T. B. 2000, ApJ, 538, L7

Seaton, M. J., Yan, Y., Mihalas, D., \& Pradhan, A. K. 1994, MNRAS, 266, 805 Sion, E. M., \& Downes, R. A. 1992, ApJ, 396, L79

Werner, K., \& Heber, U. 1992, in Atmospheres of Early-Type Stars, ed. U. Heber, \& C. S. Jeffery, Lecture Notes in Physics, 401 (Berlin: Springer), 291

Werner, K., Heber, U., \& Hunger, K. 1991, A\&A, 244, 437

Werner, K., Hamann, W.-R., Heber, U., et al. 1992, A\&A, 259, L69

Werner, K., Heber, U., \& Fleming, T. A. 1994, A\&A, 284, 907

Werner, K., Dreizler, S., Heber, U., et al. 1996, A\&A, 307, 860

Werner, K., Rauch, T., Reiff, E., Kruk, J. W., \& Napiwotzki, R. 2004a, A\&A, 427, 685

Werner, K., Rauch, T., Barstow, M. A., \& Kruk, J. W. 2004, A\&A, 421, 1169

Werner, K., Rauch, T., \& Kruk, J. W. 2005, A\&A, 433, 641

Werner, K., Rauch, T., \& Kruk, J. W. 2007, A\&A, 466, 317

Zuckerman, B., \& Reid, I. N. 1998, ApJ, 505, L143 